\title{
Epidemiology of Neonatal Septicemia in the Era of Extended Spectrum Beta-Lactamase Producing Bacteria: A Prospective Study in a Tertiary Referral Hospital
}

\author{
Dina M. Hassan ${ }^{1 *}$ (D), Lamiaa A. Madkour ${ }^{2}$ and Walaa A. Abuelhamd ${ }^{3}$ (i) \\ ${ }^{1}$ Clinical and Chemical Pathology Department, Faculty of Medicine, Cairo University, Egypt. ${ }^{2}$ Medical \\ Microbiology and Immunology Department, Faculty of Medicine, Cairo University, Egypt. ${ }^{3}$ Pediatrics and \\ Neonatology Department, Faculty of Medicine, Cairo University, Egypt
}

\begin{abstract}
A surge of extended spectrum beta lactamase (ESBL)-producers is being witnessed in the neonatal intensive care units (NICUs). Hence, the present study was conducted to analyze both the bacteriological profile and clinical outcome of neonatal septicemia, and to identify the prevalence and sensitivity of the incriminated pathogens with emphasis on ESBL producers. We conducted this study in the NICU of a tertiary referral hospital over a one-year-period. All neonates with a clinical diagnosis of sepsis (371 participants) were enrolled. Blood cultures were performed, and subsequent cultures of various specimens were done according to clinical suspicion. Antibiotic susceptibility tests were carried out and the neonates were followed up until discharge. Out of the 371 neonates, 137 (37\%) had positive blood culture results, of whom $49 \%$ died versus only $7.7 \%$ of neonates with a negative blood culture (P-value< 0.0001). Low birth weight, prematurity, and the duration of hospital stay were considered as positive blood culture risk factors. Meanwhile, among 85 cultures that yielded Gram-negative pathogens, 16 isolates were identified as ESBL producers with Klebsiella pneumoniae being the most frequently encountered isolate (19.7\%). Of the neonates inflicted with ESBL-sepsis, $62.5 \%$ died versus $11.6 \%$ with non-ESBL sepsis. Judicious antibiotic stewardship together with infection control practices can hinder the spread of drug-resistant pathogens. This is especially compelling among the vulnerable population of the NICUs. Meanwhile, rapid diagnostic modalities and timely antibiotic susceptibility tests are of paramount importance to initiate appropriate therapy which can hugely impact the clinical prognosis.
\end{abstract}

Keywords: Acinetobacter, ESBL, Klebsiella, MDR, Neonatal septicemia, NICU

*Correspondence: dinamhassan12@gmail.com; 00201006911788

(Received: August 29, 2020; accepted: September 21, 2020)

Citation: Hassan DM, Madkour LA, Abuelhamd WA. Epidemiology of Neonatal Septicemia in the Era of Extended Spectrum Beta-Lactamase Producing Bacteria: A Prospective Study in a Tertiary Referral Hospital. J Pure Appl Microbiol. 2020;14(3):21892202. doi: $10.22207 /$ JPAM.14.3.60

(C) The Author(s) 2020. Open Access. This article is distributed under the terms of the Creative Commons Attribution 4.0 International License which permits unrestricted use, sharing, distribution, and reproduction in any medium, provided you give appropriate credit to the original author(s) and the source, provide a link to the Creative Commons license, and indicate if changes were made. 


\section{INTRODUCTION}

Neonatal sepsis is considered among the top three causes of neonatal morbidity and mortality ${ }^{1}$ and accounts for approximately $25 \%$ of neonatal deaths around the globe ${ }^{2}$. In the presence of minimal warning signs, the diagnosis and treatment of sepsis pose a tedious predicament to health care givers in the neonatal intensive care units (NICUs) ${ }^{3}$. Consequently, surveillance has become vital to determine the neonatal sepsis related risk factors, as well as to identify the incriminated pathogens and their antibiotic sensitivity patterns ${ }^{4}$.

Nonetheless, the problem has been aggravated by the escalation of multidrugresistant (MDR) microorganisms which constitute a menace to the debilitated newborn $s^{5}$ thus limiting treatment options and delaying effective therapy ${ }^{6}$. Notably, extended-spectrum beta-lactamase (ESBL) producers have become the most frequent factor of neonatal sepsis in many countries ${ }^{7}$. In the meantime, antibiotics active against ESBLproducing pathogens may not be available in most settings, and even when procured, are often too expensive ${ }^{8}$.

Hence, this study aimed to identify risk factors of neonatal sepsis in the NICUs of a tertiary care hospital. We also endeavored to detect the causative bacterial pathogens and their susceptibility patterns, including the prevalence of ESBL producers and their impact on the neonate's clinical outcome.

\section{MATERIALS AND METHODS}

This is a cross sectional analytical study that has been carried out on neonates admitted to the NICU of Al-Mounira Pediatric Hospital, Cairo University, in the period from May 2016 throughout April 2017.

\section{Compliance with Ethical consideration}

Prior to commencing the current study, the Ethical Committee of the Pediatrics and Neonatology Department, Cairo University has approved our protocol. For each enrolled neonate, an informed consent was procured from the parents/guardians.

Inclusion criteria

All neonates (aged $\leq 28$ days) admitted to the NICU during the one-year-study period (371 participants) were enrolled in this study, while infants aged $>28$ days upon admission were excluded. Clinical history taking

All enrolled neonates underwent detailed history taking as follows: prenatal history including maternal medical disorders during pregnancy, maternal medications, maternal nutrition, antenatal care, premature rupture of membranes (PROM), chorioamnionitis, prolonged rupture of membranes, and history of recurrent abortion.

- Natal history including the mode of delivery, multiple pregnancy, Apgar score at 1 and 5 minutes, birth asphyxia and the presence of meconium. - Postnatal history including birth weight, sex, gestational age, feeding type (breast, artificial, total parenteral nutrition [TPN ] and its duration), admission diagnosis, age at admission, presence of central venous catheterization (including umbilical venous catheterization and jugular venous catheterization) and its duration, chest tube and its duration or continuous positive airway pressure (CPAP) use, duration of mechanical ventilation, antibiotics usage (types and duration) before collecting clinical specimens, duration of hospital stay, the use of $\mathrm{H} 2$-blockers or proton pump inhibitors, and the clinical outcome.

\section{Clinical examination of the newborn}

All newborns were exposed to full clinical examination including: recording of the anthropometric measures (weight, height, and head circumference). Systemic examination including cardiovascular, chest, and abdominal examination. Meanwhile, Ballard score was used to assess the gestational age.

\section{Laboratory investigations including}

For all participants, routine complete blood count $(\mathrm{CBC})$ and blood culture were performed on admission. The subsequent CBCs and cultures of various clinical specimens were done according to the clinical circumstances (pus/ urine/ blood/ stools/ CSF/ tips of peripheral long line catheters /tips of central venous catheters /tips of endotracheal tubes (ETT)/ bronchoalveolar lavage (BAL) fluids /tracheal aspirates / fluids of pleural taps /fluids of pericardial tap/ fluids of ascitic taps).

All recruited specimens underwent the traditional microbiological techniques concerning identification and isolation of the pathogens; 
determination of antibiotic susceptibility patterns, and detection of ESBL production.

Participants were classified according to the results of blood culture into neonates with positive blood culture and neonates with negative blood culture.

Gram negative sepsis was further classified according to the presence of ESBLproducers into ESBL sepsis and non-ESBL sepsis. Bacterial identification:

Blood specimens were cultivated onto Bactec blood culture bottles and subcultured onto blood, chocolate and MacConkey agar plates (Oxoid Co. England).

Urine specimens were cultured onto CLED (Oxoid Co. England), while sputum, tracheal aspirates, BAL, tips of peripheral long line catheters, tips of central venous catheters, tips of ETT were all cultured onto blood, chocolate and MacConkey agar plates (Oxoid Co. England).

All plates were aerobically incubated for 24 hours at the temperature of $37^{\circ} \mathrm{C}$. Identification of the bacterial isolates was carried out according to conventional microbiologic tools?.

\section{Antimicrobial Sensitivity testing}

For all isolates, this was carried out via Kirby-Bauer disc diffusion method and was interpreted in conformity with the Clinical Laboratory Standards Institute (CLSI) recommendations. ${ }^{10}$ Quality control was performed as specified by the CLSI with strains of American Type Culture Collection (ATCC) being the control.

Screening for ESBL among the isolated Gram negative bacteria

To perform the double disc synergy test (DDST), the following discs (Oxoid Co. England) were employed:

- Cefotaxime (CTX) $30 \mu \mathrm{g}$

- Ceftazidime (CAZ) $30 \mu \mathrm{g}$

- Cefpodoxime (CPD) $30 \mu \mathrm{g}$

- Ceftriaxone (CRO) $30 \mu \mathrm{g}$

- Aztreonam (ATM) $30 \mu \mathrm{g}$

- AMC (amoxicillin $20 \mu \mathrm{g}$ and clavulanic acid; CLA $10 \mu \mathrm{g})$.

The distance was adjusted to be 20 millimeters (from center to center) between each two adjacent discs. This was followed by an overnight incubation at the temperature of $37^{\circ} \mathrm{C}$.

An extension that was readily visible from the edge of the inhibition zone around any disc towards that of the amoxicillin clavulanate disc was considered positive for CLA synergy ${ }^{11}$.

Phenotypic confirmatory test for ESBLs in Gramnegative isolates

Cephalosporin/clavulanate combination disc method was applied. A half McFarland suspension was prepared from each isolate and then inoculated onto a 10-mm Mueller-Hinton agar plate, while employing ceftazidime disc $(30 \mathrm{~g})$, ceftazidime /clavulanate disc $(30 / 10 \mu \mathrm{g})$, cefepime disc (30 g), and cefepime /clavulanate disc (30/10 $\mu \mathrm{g})$. After incubation, the inhibition zone around each of the discs was measured. ESBL production was confirmed by an enlargement of over $5 \mathrm{~mm}$ in the zone diameter around any antimicrobial drug tested combined with clavulanate compared to the zone diameter around that drug alone ${ }^{12}$.

\section{Statistical Analysis}

Following coding, data were entered into the statistical package for the Social Sciences $\left(18^{\text {th }}\right.$ version). The results were depicted as diagrams and tables and then interpreted.

For all variables that are quantitative; the mean, the range, and the standard deviation were employed; and for variables that are categorical, frequencies and percentages were employed. To compare categorical data, Chi square $(\chi 2)$ test was employed.

To compare different groups, the $t$-test was applied. Z-test was used to examine the statistical significance of the estimated relative risk. To compare intergroup difference regarding nominal data, Fisher's exact test was employed. $P$-values were regarded as statistically significant if they were below or equal to 0.05 .

\section{RESULTS}

The current cross-sectional study was conducted on all neonates (371 participants) admitted at the NICU of Al-Mounira Pediatric University Hospital from May 2016 throughout April 2017.

The demographic and clinical criteria of the enrolled participants are outlined in Table (1). Meanwhile, a statistically notable difference was revealed between neonates with positive blood culture and those with negative blood culture regarding birth weight; neonates with lower birth weight being more probable to 
yield positive results of blood culture $(P$ - value $=$ $0.005)$. Other risk determinants of sepsis with a positive blood culture included prematurity, meconium staining and the duration of hospital stay (Table 2).

Table 1. Demographic, clinical data and outcome of the enrolled neonates ( $N=371$ participants)

\begin{tabular}{|c|c|c|}
\hline Variable & & Participants \\
\hline Gender & Male & $76(20.5 \%)$ \\
\hline Birth weight (gm) & Range & $1100-4600$ \\
\hline & Mean \pm SD & $2156.35 \pm 670.40$ \\
\hline Mode of delivery & NVD & $80(21.6 \%)$ \\
\hline Gestational age (weeks) & Range & $30-40$ \\
\hline & Mean \pm SD & $35.81 \pm 1.73$ \\
\hline PROM & Yes & $114(30.7 \%)$ \\
\hline & No & 257 (69.3\%) \\
\hline Meconium staining & Yes & $8(2.2 \%)$ \\
\hline & No & $363(97.8 \%)$ \\
\hline Duration of admission (days) & Range & $2-48$ \\
\hline & Mean \pm SD & $4.48 \pm 2.31$ \\
\hline Central venous catheterization & Yes & $68(18.3 \%)$ \\
\hline & No & $303(81.7 \%)$ \\
\hline Duration of central venous & Range & $3-17$ \\
\hline catheterization (days) & Mean \pm SD & $3.60 \pm 0.705$ \\
\hline Chest tube duration (days) & Range & $4-11$ \\
\hline & Mean \pm SD & $5.9 \pm 1.71$ \\
\hline TPN & Yes & $68(18.3 \%)$ \\
\hline & No & $303(81.7 \%)$ \\
\hline TPN duration (days) & Range & $3-35$ \\
\hline & Mean \pm SD & $5.90 \pm 2.21$ \\
\hline Variable & Participants & \\
\hline$N(\%)=371(100 \%)$ & & \\
\hline CPAP & Yes & $319(86 \%)$ \\
\hline & No & $52(14 \%)$ \\
\hline Duration of CPAP (days) & Range & $1-7$ \\
\hline & Mean \pm SD & $1.60 \pm 1.10$ \\
\hline Mechanical & & \\
\hline Ventilation & Yes & $316(85.2 \%)$ \\
\hline & No & $55(14.8 \%)$ \\
\hline Duration of mechanical & Range & $1-30$ \\
\hline ventilation (days) & Mean \pm SD & $3.5 \pm 1.30$ \\
\hline Duration of oxygen support & Range & $2-38$ \\
\hline (days) & Mean \pm SD & $7.54 \pm 4.50$ \\
\hline ETT & Yes & $316(85.2 \%)$ \\
\hline & No & $55(14.8 \%)$ \\
\hline Duration of ETT (days) & Range & $1-30$ \\
\hline & Mean \pm SD & $3.5 \pm 1.30$ \\
\hline Clinical outcome & Died & $85(23 \%)$ \\
\hline & Survived & $286(77 \%)$ \\
\hline
\end{tabular}

NVD = normal vaginal delivery; $\mathrm{PROM}=$ premature rupture of membranes; $\mathrm{TPN}=$ total parenteral nutrition; $\mathrm{CPAP}=$ continuous positive airway pressure; ETT= endotracheal tube.

Journal of Pure and Applied Microbiology logistic regression analysis, it was revealed that the duration of hospital stay was the only independent predictor of a positive blood culture 
Table 2. Risk factors of sepsis among the studied neonates $(\mathrm{N}=371)$

\begin{tabular}{|c|c|c|c|c|c|}
\hline \multicolumn{2}{|c|}{ Potential risk factors } & \multirow{2}{*}{ 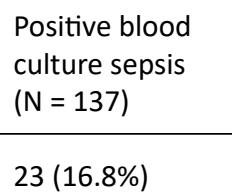 } & \multirow{2}{*}{$\begin{array}{l}\text { Negative blood } \\
\text { culture sepsis } \\
(\mathrm{N}=234)\end{array}$} & \multirow{2}{*}{$\begin{array}{l}\text { P-value } \\
0.177\end{array}$} & \multirow{2}{*}{$\begin{array}{l}\text { RR } \\
(95 \% \mathrm{Cl})\end{array}$} \\
\hline Gender & Male & & & & \\
\hline Birthweight & Mean $\pm S D$ & $2029.42 \pm 674.46$ & $2230.66 \pm 658.15$ & 0.005 & ------ \\
\hline (gm) & $\geq 2500 \mathrm{gm}$ & $44(32.2 \%)$ & $51(21.8)$ & 0.022 & 0.728 \\
\hline & $<2500 \mathrm{gm}$ & $93(67.8 \%)$ & $183(78.2 \%)$ & & $\begin{array}{l}(0.554 \text { to } \\
0.955)\end{array}$ \\
\hline \multirow{3}{*}{$\begin{array}{l}\text { Gestational age } \\
\text { (weeks) }\end{array}$} & Mean $\pm S D$ & $35.79 \pm 1.95$ & $35.82 \pm 1.60$ & 0.858 & ------ \\
\hline & Preterm & $101(73.7 \%)$ & $130(55.6 \%)$ & 0.001 & 1.700 \\
\hline & Full term & $36(26.3 \%)$ & 104 (44.4\%) & & $\begin{array}{l}(1.238 \\
\text { to } 2.335)\end{array}$ \\
\hline Mode of delivery & NVD & $26(19.1 \%)$ & $54(23.1 \%)$ & 0.372 & ------ \\
\hline $\begin{array}{l}\text { Apgar score at } \\
5 \text { minutes }\end{array}$ & Mean $\pm S D$ & $7.39 \pm 0.893$ & $7.40 \pm 0.913$ & 0.914 & ------- \\
\hline \multirow[t]{2}{*}{ PROM } & Yes & $43(31.4 \%)$ & $71(30.3 \%)$ & 0.833 & 1.031 \\
\hline & No & $94(68.6 \%)$ & $163(69.7 \%)$ & & $\begin{array}{l}(0.775 \text { to } \\
1.372)\end{array}$ \\
\hline \multirow[t]{2}{*}{ Meconium staining } & Yes & $7(5.1 \%)$ & $1(0.4 \%)$ & 0.003 & 2.443 \\
\hline & No & 130 (94.9\%) & 233 (99.6\%) & & $\begin{array}{l}(1.817 \text { to } \\
3.285)\end{array}$ \\
\hline $\begin{array}{l}\text { Duration of } \\
\text { admission (days) }\end{array}$ & Mean $\pm S D$ & $5.24 \pm 3.43$ & $3.72 \pm 1.19$ & $<0.0001$ & ------ \\
\hline Central venous & Yes & $12(8.8 \%)$ & $56(23.9 \%)$ & 0.000 & 0.428 \\
\hline Catheterization & No & $125(91.2 \%)$ & $178(76.1 \%)$ & & $\begin{array}{l}(0.252 \text { to } \\
0.727)\end{array}$ \\
\hline $\begin{array}{l}\text { Duration of central } \\
\text { venous catheterization } \\
\text { (days) }\end{array}$ & Mean $\pm S D$ & $5.10 \pm 0.000$ & $2.10 \pm 1.41$ & $<0.0001$ & ----- \\
\hline $\begin{array}{l}\text { Duration of chest } \\
\text { tube (days) }\end{array}$ & Mean $\pm S D$ & $6.3 \pm 3.2$ & $6.6 \pm 3.8$ & 0.92 & ----- \\
\hline \multirow[t]{2}{*}{ TPN } & Yes & $12(8.8 \%)$ & $56(23.9 \%)$ & 0.000 & 0.428 \\
\hline & No & 125 (91.2\%) & $178(76.1 \%)$ & & $\begin{array}{l}(0.252 \text { to } \\
0.727)\end{array}$ \\
\hline $\begin{array}{l}\text { TPN } \\
\text { duration (days) }\end{array}$ & Mean $\pm S D$ & $6.80 \pm 3.42$ & $5.0 \pm 1.0$ & $<0.0001$ & ----- \\
\hline \multirow[t]{2}{*}{ CPAP } & Yes & $118(86.1 \%)$ & $201(86 \%)$ & 0.950 & 1.012 \\
\hline & No & 19 (13.9\%) & $33(14 \%)$ & & $\begin{array}{l}(0.688 \\
\text { to } 1.489)\end{array}$ \\
\hline $\begin{array}{l}\text { Duration of CPAP } \\
\text { (days) }\end{array}$ & Mean $\pm S D$ & $1.51 \pm 0.870$ & $1.69 \pm 1.32$ & 0.492 & ------ \\
\hline Mechanical ventilation & & $116(85 \%)$ & $200(85.5 \%)$ & 0.833 & 0.961 \\
\hline Yes & & No & $21(15 \%)$ & $34(14.5 \%)$ & $\begin{array}{l}(0.667 \text { to } \\
1.387)\end{array}$ \\
\hline $\begin{array}{l}\text { Duration of mechanical } \\
\text { ventilation (days) }\end{array}$ & Mean $\pm S D$ & $5.00 \pm 1.1$ & $2.00 \pm 1.41$ & $<0.0001$ & ------ \\
\hline $\begin{array}{l}\text { Duration of oxygen } \\
\text { support (days) }\end{array}$ & Mean $\pm S D$ & $7.27 \pm 3.35$ & $7.82 \pm 5.80$ & 0.307 & ----- \\
\hline \multirow[t]{2}{*}{ ETT } & Yes & $116(85 \%)$ & $200(85.5 \%)$ & 0.833 & 0.961 \\
\hline & No & $21(15 \%)$ & $34(14.5 \%)$ & & $\begin{array}{l}(0.667 \\
\text { to } 1.387)\end{array}$ \\
\hline ETT duration(days) & Mean $\pm S D$ & $5.00 \pm 1.1$ & $2.00 \pm 1.41$ & $<0.0001$ & ----- \\
\hline
\end{tabular}


Table 2. Cont...

\begin{tabular}{|c|c|c|c|c|c|}
\hline \multicolumn{2}{|c|}{ Potential risk factors } & \multirow{2}{*}{ 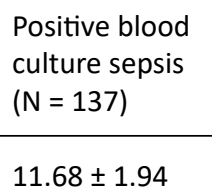 } & \multirow{2}{*}{ 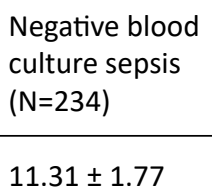 } & \multirow{2}{*}{$\begin{array}{l}\text { P-value } \\
0.064\end{array}$} & \multirow{2}{*}{$\begin{array}{l}\text { RR } \\
\begin{array}{l}(95 \% \mathrm{Cl}) \\
----\end{array}\end{array}$} \\
\hline $\mathrm{HB}$ & Mean $\pm S D$ & & & & \\
\hline HCT & Mean $\pm S D$ & $33.78 \pm 6.18$ & $32.80 \pm 5.17$ & 0.104 & ---- \\
\hline PLT & Mean $\pm S D$ & $271.40 \pm 108.26$ & $283.32 \pm 110.56$ & 0.313 & ----- \\
\hline TLC & Mean $\pm S D$ & $15.52 \pm 9.79$ & $15.79 \pm 6.84$ & 0.753 & ---- \\
\hline \multirow[t]{2}{*}{ Outcome } & Died & 67 (49\%) & $18(7.7 \%)$ & $<0.0001$ & ---- \\
\hline & Survived & 70 (51\%) & $216(92.3 \%)$ & & \\
\hline
\end{tabular}

$\mathrm{RR}=$ relative risk; $95 \% \mathrm{Cl}=95 \%$ confidence interval; $\mathrm{NVD}=$ normal vaginal delivery; $\mathrm{PROM}=$ premature rupture of membrane TPN=total parenteral nutrition; $\mathrm{ETT}=$ endotracheal tube; $\mathrm{CPAP}=$ continuous positive airway pressure; $\mathrm{HB}=$ hemoglobin; $\mathrm{HCT}=$ hematocrit; PLT= platelet; TLC = total leukocytic count.

Table 3. Multivariable binary logistic regression analysis of independent predictors of positive blood culture

\begin{tabular}{lcccccc}
\hline Variable & $\begin{array}{c}\text { Regression } \\
\text { coefficient }\end{array}$ & $\begin{array}{c}\text { Standard } \\
\text { error }\end{array}$ & $\begin{array}{c}\text { Wald } \\
\text { statistic }\end{array}$ & P-value & $\begin{array}{c}\text { Odds } \\
\text { ratio }\end{array}$ & $\begin{array}{c}95 \% \mathrm{Cl} \text { of } \\
\text { odds ratio }\end{array}$ \\
\hline $\begin{array}{l}\text { Duration of hospital } \\
\text { stay (days) }\end{array}$ & -0.171 & 0.047 & 13.180 & $<0.001$ & 0.843 & 0.769 to 0.924 \\
$\begin{array}{l}\text { Duration of TPN (days) } \\
\text { Duration of MV (days) }\end{array}$ & -6.252 & 939.471 & 0.000 & 0.995 & 0.002 & 0.00 to infinity \\
Meconium staining & -2.077 & 0.073 & 3.307 & 0.069 & 1.141 & 0.990 to 01.316 \\
Constant & 2.849 & 1.091 & 3.626 & 0.057 & 0.125 & 0.015 to 1.063 \\
\hline
\end{tabular}

Table 4. Pathogens isolated from blood cultures of the enrolled neonates and their susceptibility patterns

\begin{tabular}{|c|c|c|}
\hline Pathogen & $\begin{array}{l}N(\%) \\
\text { Total = } 371 \\
(100 \%)\end{array}$ & $\begin{array}{l}\text { Antibiotic(s) to which the } \\
\text { highest percentage of } \\
\text { isolates was susceptible }\end{array}$ \\
\hline Klebsiella spp. & 27 (19.7\%) & Polymyxin (81\%) \\
\hline Acinetobacter spp. & $21(15.3 \%)$ & Polymyxin (71\%) \\
\hline E. coli & 19 (13.9\%) & Polymyxin (68\%) \\
\hline Staph. aureus (MSSA) & $18(13.1 \%)$ & Vancomycin (46\%) \\
\hline CoNS & $16(11.7 \%)$ & Linezolid, vancomycin (77\%) \\
\hline MRSA & $12(8.8 \%)$ & Rifampicin, linezolid (88\%) \\
\hline Strept. Pneumoniae & $7(5.1 \%)$ & Linezolid, vancomycin (100\%) \\
\hline Pseudomonas spp. & $6(4.4 \%)$ & Levofloxacin (81\%) \\
\hline Enterobacter spp. & $3(2.2 \%)$ & Amoxicillin-clavulanate (77\%) \\
\hline
\end{tabular}

MSSA = methicillin-sensitive Staphylococcus aureus; CoNS = Coagulase-negative Staphylococcus; E.coli= Escherichia coli; MRSA= methicillin-resistant Staphylococcus aureus.

result $(\mathrm{P}$ - value $=<0.001 ;$ adjusted odds ratio $=$ 0.843) (Table 3).

Among the pathogens isolated from blood cultures, Klebsiella spp. was the most frequently encountered (19.7\%), and $81 \%$ of the Klebsiella isolates were polymyxin-susceptible (Table 4).
Out of 85 culture specimens that yielded Gram-negative bacteria, 16 of the retrieved isolates were proven to be ESBL producers (Figure 1).

In the meantime, the relative risk (RR) for neonates with ESBL was calculated and showed no statistically significant association between 
exposure to any of the risk factors and the occurrence of ESBL sepsis. All estimated RRs had $95 \%$ confidence intervals $(\mathrm{Cl})$ that included the null value of $R R=1$ (Table 5).

Employing the combination disc method, 16 isolates out of $85 \mathrm{Gram}$-negative isolates were identified as ESBL-producers (18.8\%), of which 11 isolates were also detected as ESBL producers by the DDST (Table 6). A good agreement was found between the combination disc method and the DDST (Kappa value $=0.710$ ). With the combination

Table 5. Comparison between ESBL sepsis and with non-ESBL sepsis regarding the potential risk factors

\begin{tabular}{|c|c|c|c|c|c|}
\hline \multicolumn{2}{|c|}{ Potential risk factors } & \multirow{2}{*}{$\begin{array}{l}\text { ESBL sepsis } \\
\mathrm{N}(\%)=16(100 \%) \\
0(0 \%)\end{array}$} & \multirow{2}{*}{$\begin{array}{l}\text { Non-ESBL sepsis } \\
\text { N }(\%)=69(100 \%) \\
16(23.2 \%)\end{array}$} & \multirow{2}{*}{$\begin{array}{l}\text { P- value } \\
0.346\end{array}$} & \multirow{2}{*}{$\begin{array}{l}\begin{array}{l}\mathrm{RR} \\
(95 \% \mathrm{Cl})\end{array} \\
---\end{array}$} \\
\hline Gender & Male & & & & \\
\hline & Female & $16(100.0 \%)$ & $53(76.8 \%)$ & & \\
\hline \multirow[t]{3}{*}{ Birth weight (gm) } & Mean $\pm S D$ & $2111.88 \pm 876.20$ & $2075.51 \pm 704.30$ & 0.588 & --- \\
\hline & $\geq 2500 \mathrm{gm}$ & $4(25 \%)$ & $29(42 \%)$ & 0.227 & 1.904 \\
\hline & $<2500 \mathrm{gm}$ & $12(75 \%)$ & $40(85 \%)$ & & $\begin{array}{l}(0.670 \\
\text { to } 5.409)\end{array}$ \\
\hline \multirow{3}{*}{$\begin{array}{l}\text { Gestational age } \\
\text { (weeks) }\end{array}$} & Mean $\pm S D$ & $36.31 \pm 2.02$ & $35.95 \pm 1.78$ & 0.192 & --- \\
\hline & Preterm & 11 (68.8\%) & $35(50.7 \%)$ & 0.207 & 1.865 \\
\hline & Full term & $5(31.3 \%)$ & $34(49.3 \%)$ & & $\begin{array}{l}(0.709 \text { to } \\
4.907)\end{array}$ \\
\hline Mode of delivery & NVD & $3(18.8 \%)$ & $19(27.5 \%)$ & 0.002 & ---- \\
\hline \multirow[t]{2}{*}{ PROM } & Yes & $6(37.5 \%)$ & $21(30.4 \%)$ & 0.584 & 1.289 \\
\hline & No & $10(62.5 \%)$ & $48(69.6 \%)$ & & $\begin{array}{l}(0.522 \text { to } \\
3.181)\end{array}$ \\
\hline Apgar score (1minute) & Mean $\pm S D$ & $5.06 \pm 0.854$ & $5.06 \pm 0.833$ & 0.898 & ----- \\
\hline Apgar score (5 minutes) & Mean $\pm S D$ & $7.75 \pm 0.931$ & $7.41 \pm 0.929$ & 0.342 & ---- \\
\hline Meconium & Yes & $1(6.3 \%)$ & $3(4.3 \%)$ & 0.396 & 1.350 \\
\hline staining & No & $15(93.8 \%)$ & $66(95.7 \%)$ & & $\begin{array}{l}(0.233 \text { to } \\
7.830)\end{array}$ \\
\hline \multirow[t]{2}{*}{ TPN } & Yes & $1(6.3 \%)$ & $4(5.8 \%)$ & 0.346 & 1.067 \\
\hline & No & 15 (93.8\%) & $65(94.2 \%)$ & & $\begin{array}{l}(0.174 \\
\text { to } 6.527)\end{array}$ \\
\hline TPN duration (days) & Mean $\pm S D$ & $5.00 \pm 1.00$ & $5.40 \pm 1.45$ & 0.081 & ----- \\
\hline \multirow{2}{*}{ CPAP } & Yes & $15(93.8 \%)$ & $59(86 \%)$ & 0.414 & 2.230 \\
\hline & No & $1(6.3 \%)$ & $10(14.5 \%)$ & & $\begin{array}{l}(0.326 \text { to } \\
15.249)\end{array}$ \\
\hline $\begin{array}{l}\text { Duration of CPAP } \\
\text { (days) }\end{array}$ & Mean $\pm S D$ & $1.17 \pm 0.408$ & $3.48 \pm 2.87$ & 0.760 & ---- \\
\hline \multirow{3}{*}{$\begin{array}{l}\text { Mechanical } \\
\text { ventilation }\end{array}$} & $10(63 \%)$ & $56(81.2 \%)$ & 0.100 & 0.480 & (0.200 to \\
\hline & Yes & & & & 1.150) \\
\hline & No & $6(37.5 \%)$ & $13(18.8 \%)$ & & \\
\hline $\begin{array}{l}\text { Duration of mechanical } \\
\text { ventilation (days) }\end{array}$ & Mean $\pm S D$ & $3.67 \pm 1.96$ & $5.75 \pm 2.80$ & 0.061 & ----- \\
\hline $\begin{array}{l}\text { Duration of oxygen } \\
\text { support (days) }\end{array}$ & Mean $\pm S D$ & $5.63 \pm 2.57$ & $8.90 \pm 5.43$ & 0.216 & ----- \\
\hline $\mathrm{HB}$ & Mean $\pm S D$ & $11.31 \pm 1.94$ & $11.45 \pm 1.83$ & 0.620 & ----- \\
\hline HCT & Mean $\pm S D$ & $32.66 \pm 6.64$ & $32.96 \pm 5.87$ & 0.931 & ----- \\
\hline PLT & Mean $\pm S D$ & $338.19 \pm 138.97$ & $269.99 \pm 115.97$ & 0.693 & ----- \\
\hline TLC & Mean $\pm S D$ & $14.43 \pm 4.27$ & $14.67 \pm 5.095$ & 0.822 & ------ \\
\hline
\end{tabular}

$\mathrm{RR}=$ relative risk; $\mathrm{Cl}=$ confidence interval; $\mathrm{NVD}=$ normal vaginal delivery; $\mathrm{PROM}=$ premature rupture of membranes; $\mathrm{TPN}=$ total parenteral nutrition; $\mathrm{CPAP}=$ continuous positive airway pressure; $\mathrm{HB}=$ hemoglobin; $\mathrm{HCT}=$ hematocrit; $\mathrm{PLT}=$ platelet count; $\mathrm{TLC}=$ total leukocytic count. 
disc method applied as a gold standard, the DDST was found to be $91.96 \%$ accurate (Table 7 ).

The commonest ESBL-producing organism isolated from cultures was Klebsiella spp., while
Acinetobacter spp. was the commonest organism among non-ESBL producers. It's worthwhile to mention that $62.5 \%$ of the neonates with ESBLsepsis died versus $11.6 \%$ of neonates with non-

Table 6. Comparison between the combination disc method and the DDST regarding ESBL detection

\begin{tabular}{|c|c|c|c|c|c|}
\hline & & & \multicolumn{2}{|c|}{ DDST } & \multirow[t]{2}{*}{ Total } \\
\hline & & & ESBL & Non-ESBL & \\
\hline \multirow{4}{*}{$\begin{array}{l}\text { Combined disk } \\
\text { method }\end{array}$} & ESBL & Count & 11 & 5 & 16 \\
\hline & & $\%$ within DDST & $84.6 \%$ & $6.9 \%$ & $18.8 \%$ \\
\hline & Non-ESBL & Count & 2 & 67 & 69 \\
\hline & & $\%$ within DDST & $15.4 \%$ & $93.1 \%$ & $81.2 \%$ \\
\hline \multirow[t]{2}{*}{ Total } & & Count & 13 & 72 & 85 \\
\hline & & $\%$ within DDST & $100 \%$ & $100 \%$ & $100 \%$ \\
\hline
\end{tabular}

Table 7. Performance of the DDST compared to the combination disc method as a gold standard

\begin{tabular}{lccccc}
\hline Item & Sensitivity & Specificity & $\begin{array}{c}\text { Positive } \\
\text { predictive } \\
\text { value (PPV) }\end{array}$ & $\begin{array}{c}\text { Negative } \\
\text { predictive } \\
\text { value (NPV) }\end{array}$ & Accuracy \\
\hline DDST & $84.6 \%$ & $93.1 \%$ & $68.7 \%$ & $97.1 \%$ & $91.96 \%$ \\
\hline
\end{tabular}

Table 8. Comparison between ESBL and non-ESBL producing organisms according to specimen, retrieved isolates, and clinical outcome

\begin{tabular}{|c|c|c|}
\hline & $\mathrm{ESBL}(\mathrm{N}=16)$ & Non-ESBL $(N=69)$ \\
\hline Specimen & - Blood $(\mathrm{N}=16)$ & $\begin{array}{l}\text { - } \text { Blood }(\mathrm{N}=60) \\
\text { - ETT aspirate }(\mathrm{N}=2) \\
\text { - Sputum }(\mathrm{N}=3) \\
\text { - Wound swab }(\mathrm{N}=1) \\
\text { - Urine }(\mathrm{N}=3)\end{array}$ \\
\hline Organism N (\%) & $\begin{array}{l}\text { - Blood culture } \\
\text { Klebsiella: } 12(75 \%) \\
\text { E. coli: } 2(12.5 \%) \\
\text { Acinetobacter: } 2(12.5 \%)\end{array}$ & $\begin{array}{l}\text { - Blood culture } \\
\text { Acinetobacter: } 17(24.6 \%) \\
\text { Klebsiella: } 15(21.7 \%) \\
\text { E. coli: } 13(18.8 \%) \\
\text { Pseudomonas: } 9(13 \%) \\
\text { Enterobacter: } 6(8.7 \%) \\
\text { - ETT culture: } \\
\text { Klebsiella: } 2 \text { (2.9\%) } \\
\text { - Sputum culture: } \\
\text { Acinetobacter: } 3(4.3 \%) \\
\text { - Wound culture } \\
\text { Pseudomonas: } 1(1.4 \%) \\
\text { - Urine culture } \\
\text { Pseudomonas: } 2(2.8 \%) \\
\text { E. coli: } 1(1.4 \%)\end{array}$ \\
\hline Clinical outcome & & \\
\hline $\begin{array}{l}\text { - Died } \\
\text { - Survived } \\
\text { - P-value }\end{array}$ & $\begin{array}{l}10(62.5 \%) \\
6(37.5 \%) \\
\end{array}$ & $\begin{array}{l}8(11.6 \%) \\
61(88.4 \%)\end{array}$ \\
\hline
\end{tabular}


ESBL sepsis, revealing a statistically significant difference (P-value $<0.0001$ ) (Table 8).

Regarding the susceptibility pattern of the isolated pathogens, Klebsiella spp. revealed $100 \%$ resistance to amoxicillin- clavulanate, ceftazidime, as well as to cefepime; while Acinetobacter spp. demonstrated $100 \%$ resistance to amoxicillinclavulanate and ampicillin- sulbactam (Figure 2).

\section{DISCUSSION}

The WHO has evaluated that 1.6 million deaths happen universally due to neonatal infections and $40 \%$ of them happen in developing countries $^{13}$. Neonatal sepsis, a life-threatening condition, requires quick antimicrobial therapy, and it is significant to choose an antibiotic combination that covers the foremost common pathogens ${ }^{14}$. Blood culture has remained the gold standard of diagnosis, despite its low sensitivity which may be ascribed to little volume of the blood sample, or empirical antibiotics prescription earlier to sampling ${ }^{15}$.

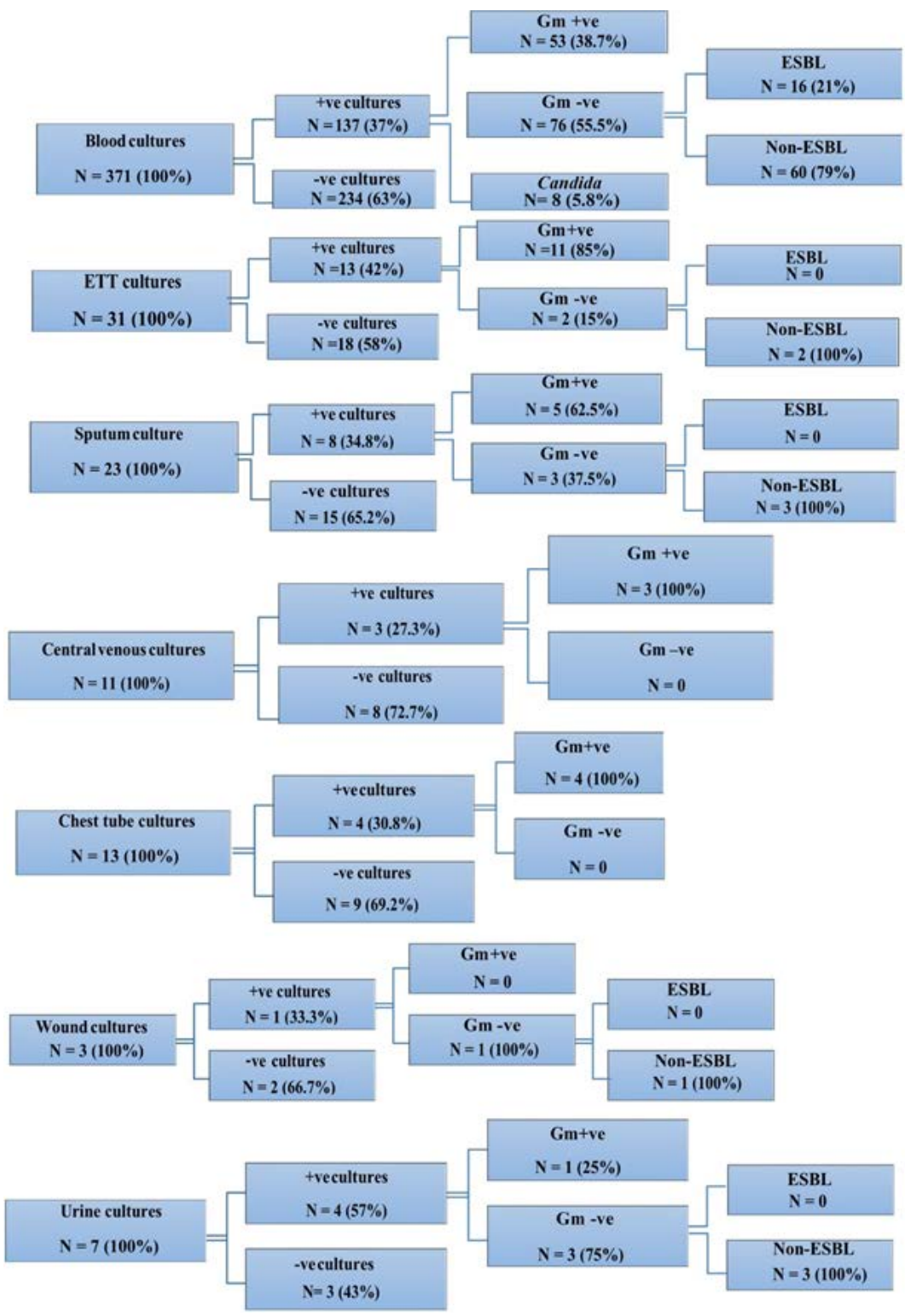

Fig. 1. Outcome of cultures and ESBL detection tests in the studied neonates 
In the present study, we investigated 371 neonates admitted to the NICU of a tertiary care hospital with a clinical suspicion of septicemia.

The rate of blood culture demonstrated that sepsis among the neonates was $37 \%(137 / 371$ cases). Comparative rates were detailed in other countries counting Tanzania $(39 \%)^{16}$ and Cameroon $(34.7 \%)^{17}$.

In the meantime, a few research depicted higher frequencies of $46 \%$ and $60.4 \%$ in India [18], where quarter of the worldwide neonatal mortality happens ${ }^{4}$.
In the meantime, our study demonstrated that Klebsiella pneumoniae was the foremost common isolated organism at the NICU followed by Acinetobacter spp. Likewise, a study from India reported that Klebsiella spp. was the leading pathogen in the $\mathrm{NICU}^{19}$.

Nonetheless, a study from Canada reported that Pseudomonas aeruginosa was the most common isolated organism followed by Klebsiella and Acinetobacter ${ }^{20}$.

Apparently, the organisms causing neonatal septicemia differ from one area to

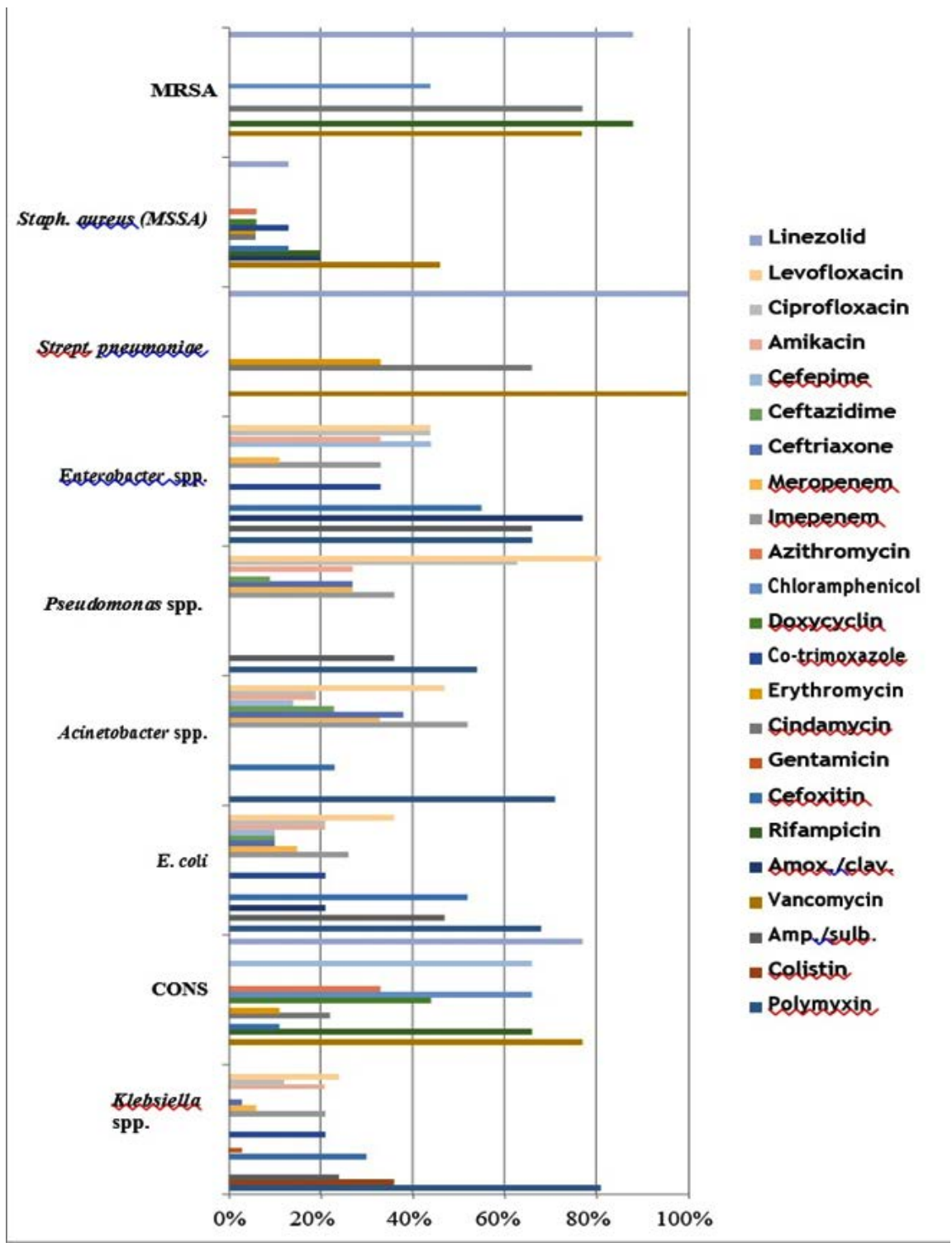

Fig. 2. Susceptibility patterns of the isolated pathogens 
another and may also change with respect to time, even in the same area ${ }^{21}$. This mandates the importance of surveillance programs and timely antibiotic sensitivity tests.

In our study, Candida was recognized in $5.8 \%$ of septic neonates, yet there is a wide variety within the detailed rate of fungal septicemia in the NICU; extending from $2.6 \%$ to $16.7 \%$ among very low birth weight infants ${ }^{22}$.

In our study, about $81 \%$ of Klebsiella spp. were polymyxin-sensitive. And indeed higher susceptibility (100\%) was found in a study from China ${ }^{23}$.

On the other hand, Klebsiella isolates in our study were $100 \%$ resistant to amoxicillinclavulanic, 3rd generation cephalosporins such as ceftazidime and 4 th generation cephalosporins such as cefepime. Additionally, most of the isolates were highly resistant to meropenem (94\%) and imipenem (79 \%).

The worrisome upward shove of MDR Gram-negative microorganisms with the relative dearth of new antibiotics has led to the revival of different drug classes as polymyxins, which are effective against $A$ cinetobacter spp., Pseudomonas aeruginosa, Klebsiella spp., and Enterobacter spp. ${ }^{24}$.

Because of the potential drawbacks of polymyxin (including neuro- and nephro-toxicity), it is generally reserved for resistant infections. It penetrates poorly into the CSF and the presence of meningeal infection would not improve its absorption ${ }^{25}$.

Moreover, polymyxins monotherapy tends to be a possible cause of hetero-resistance among patients exposed to polymyxins solely ${ }^{26}$.

Thus, an additional principle for employing combination therapy is to inhibit the hetero-resistance.

In our study, prematurity, longer period of hospital stay, mechanical ventilation, TPN and central venous catheterization had been determined to be risk factors of neonatal sepsis. Using the multivariate logistic regression analysis, the period of hospital stay has been described as an independent risk factor linked to neonatal infections.

Preterm infants are consistently highly prone to infection due to their immature host defense mechanisms and invasive life support systems. Stoll ${ }^{27}$ has mentioned a 3 - to 10 -fold higher incidence of infection than full-term normal birth weight infants.

Meanwhile, the duration of health facility stay proved to be an independent risk determinant of sepsis, suggesting that the health care surroundings are crucial factors in the transmission of pathogens. This corroborates the finding of in the past research ${ }^{28}$.

On the other hand, parenteral nutrition (PN) is increasingly being used, and often at an earlier age; for preterm neonates before full enteral feeding can be administered. There is almost universal agreement that the administration of PN is a major risk factor for neonatal sepsis ${ }^{29}$. Okada et al. ${ }^{30}$ reported that long term PN would impair host defense mechanisms and bactericidal activity. A study found that the risk of neonatal infections was about 13 times more among catheterized neonates and two times higher in those with $\mathrm{TPN}^{31}$. Another study from Taiwan revealed that the administration of PN was associated with approximately 6 -fold greater risk of blood stream infections ${ }^{32}$.

Notably, the ventilatory assist and central venous lines are the two most common invasive-treatment modalities used in NICUs. Neonates exposed to these devices are frequently at excessive threat of the prevalence of sepsis ${ }^{33}$. Infections associated with these devices can be preventable via good practice, such as aseptic techniques in insertion, surveillance and timely removal of devices as soon as they are no longer clinically required ${ }^{34}$.

In the current study, the overall mortality rate among admitted neonates was $23 \%$ (85/371 cases) with the mortality in culture-positive sepsis reaching $49 \%$ (67/137 cases) in contrast to $7.7 \%$ in culture-negative sepsis.

Such a distressingly excessive mortality may also be linked to the severity of illness at presentation, type of organism and quality of neonatal care. Meanwhile, it is additionally vital to apprehend and address the risk factors leading to this negative outcome.

ESBL-associated infections represent a significant cause of neonatal morbidity and mortality all over the world. However, the incidence of such infections varies considerably in various areas, from $37 \%$ in Latin America and 
$7 \%$ in the United States to $5-56 \%$ in a number of Asian territories ${ }^{35}$.

The existing study confirmed that out of 85 Gram-negative isolated pathogens, sixteen isolates (18.8\%) were ESBL-producers, with Klebsiella spp. being the most common organism. However, greater percentages of ESBL-producers (39.6\% and $67.3 \%$ ) had been demonstrated in other research ${ }^{18,19}$.

Such variations in the percentage of ESBL producers may be due to regional diversities. Distinct regional variations have been detected in the incidence of ESBL sepsis ${ }^{36}$.

In our study, the mortality rate in ESBLsepsis had been greater $(62.5 \%)$ compared to non-ESBL sepsis (11.6\%) and the difference was statistically significant. This finding coincides with that reported by Yusef et $\mathrm{al}^{37}$ who cited that the mortality rate with MDR-associated sepsis was $60 \%$ versus $13 \%$ with non-MDR sepsis. Nonetheless, different studies mentioned lower mortality rates of $39.1 \%{ }^{38}$ and $34.4 \%$ with ESBL sepsis. These discrepancies may additionally pertain to a number of elements like the turnaround time of blood cultures or the use of automatic assays ${ }^{5}$.

In the present study, the DDST was able to identify eleven out of 13 ESBL-producers detected by using the combination disc method, hence displaying a good agreement (Kappa=0.71) and a sensitivity of $84.6 \%$.

These findings were in line with those of Daef et al. ${ }^{39}$ who stated that 21 out of 23 (91.3\%) potentially ESBL-producing Enterobacteriaceae tested positive by using the DDST.

Worth mentioning, the DDST can also miss a few ESBL cases because of the trouble of optimal disc spacing and the proper storage of the clavulanic acid containing discs ${ }^{40}$. The CLSI therefore advocated the use of the combined disc test for the phenotypic affirmation of the ESBL producers among $E$. coli and $K$. pneumoniae ${ }^{10}$.

The worrisome evolution of MDR microorganisms points to the significance of prudent antibiotic policies in the NICU. The rise in ESBL producers may additionally be stemming from the selective pressure imposed via the extensive use of antimicrobials. Meanwhile, the various percentages of ESBL-producing isolates amongst the studies could be incidental to regional variations.
Adhering to hygienic procedures, such as hand washing, the use of sterile equipment, patient cohorting and screening of attending group of workers and mothers for MDR pathogens can help stop the spread of these resistant strains. This study stresses that antimicrobial resistance is a worldwide issue and emphasizes the need for surveillance and promotion of restrictive antibiotic policies including a tailored therapy after studying sensitivity pattern. This can halt the further pervasion of ESBL-producers and improve the prognosis of neonatal sepsis.

\section{ACKNOWLEDGMENTS}

None.

\section{CONFLICT OF INTEREST}

The authors declare that there is no conflict of interest.

\section{AUTHORS' CONTRIBUTION}

All authors listed have made a substantial, direct and intellectual contribution to the work, and approved it for publication.

\section{FUNDING}

None.

\section{ETHICS STATEMENT}

The protocol of the study has been approved by the Ethical Committee of the Pediatrics and Neonatology Department, Cairo University. An informed consent was obtained from the parents/guardians of each enrolled neonate

\section{DATA AVAILABILITY}

All datasets generated or analyzed during this study are included in the manuscript.

\section{REFERENCES}

1. Bhutta ZA, Chopra M, Axelson H, et al. Countdown to 2015 decade report (2000-10): taking stock of maternal, newborn, and child survival. Lancet. 2010;375(9730):2032-44. doi: 10.1016/S0140$6736(10) 60678-2$

2. World Health Organization (WHO) Levels and Trends in Child Mortality 2012. Available at: http://www. who.int/maternal_child_adolescent/documents/ levels_trends_child_mortality_2012/en/.

3. Sharma D, Kumar C, Pandita A, Pratap OT, Dasi T, Murki S. Bacteriological profile and clinical 
predictors of ESBL neonatal sepsis. J Matern Fetal Neonatal Med. 2016;29(4):567-70. doi: 10.3109/14767058.2015.1011118

4. Bhuvaneswari B. Bacteriological profile and antibiogram pattern of neonatal sepsis with special reference to ESBL producing Klebsiella pneumoniae in a tertiary care hospital. Univ J Pre Paraclin Sci. 2017;2(7).

5. Marando R, Seni J, Mirambo MM, et al. Predictors of the extended-spectrum-beta lactamases producing Enterobacteriaceae neonatal sepsis at a tertiary Hospital, Tanzania. Int J Med Microbiol. 2018;308(7):803-11. doi: 10.1016/j.ijmm.2018.06.012

6. Patel SJ, Saiman L. Antibiotic resistance in neonatal intensive care unit pathogens: mechanisms, clinical impact, and prevention including antibiotic stewardship. Clin Perinatol. 2010;37(3):547-563. doi: 10.1016/j.clp.2010.06.004

7. D'Andrea MM, Arena F, Pallecchi L, Rossolini GM. CTX$M$-type $\beta$-lactamases: a successful story of antibiotic resistance. Int J Med Microbiol. 2013:303(6-7):305-17. doi: 10.1016/j.ijmm.2013.02.008

8. Vandijck DM, Depaemelaere M, Labeau SO, et al. Daily cost of antimicrobial therapy in patients with intensive care unit-acquired, laboratory-confirmed bloodstream infection. Int J Antimicrob Agents. 2008;31(2):161-165. doi: 10.1016/j.ijantimicag.2007.10.015

9. Murray PR, Baron EJ. Manual of clinical microbiology. 9th ed. ASM P Washington, D.C. 2007.

10. Clinical and Laboratory Standards Institute (CLSI). Performance standards for antimicrobial susceptibility testing; twenty-sixth informational supplement. Wayne, PA: CLSI. 2016:Document M100-S26.

11. Jarlier $\mathrm{V}$, Nicolas $\mathrm{M}-\mathrm{H}$, Fournier $\mathrm{G}$ and Philippon $\mathrm{A}$. Extended board-spectrum beta-lactamases conferring transferable resistance to newer beta-lactam agents in Enterbacteriaceae: hospital Prevalence and susceptibility patterns. Rev Infect Dis. 1988;10(4):86778. doi: $10.1093 /$ clinids/10.4.867

12. Rupp ME, Fey PD. Extended spectrum beta - Lactamase (ESBL)-producing Enterbacteriaceae: Considerations for diagnosis, prevention and drug treatment. Drugs. 1996;63(4):353-365. doi: 10.2165/00003495200363040-00002

13. Zakariya BP, Bhat V, Harish BN, Babu TA, Joseph NM. Neonatal sepsis in a tertiary care hospital in South India: Bacteriological profile and antibiotic sensitivity pattern. Indian J Pediatr. 2011;78(4):413-417. doi: 10.1007/s12098-010-0314-8

14. Taheri PA, Eslamieh H, Salamati P. Is ceftizoxime an appropriate surrogate for amikacin in neonatal sepsis treatment? A randomized clinical trial. Acta Med Iran. 2011;49(8):499-503

15. Kalathia MB, Shingala PA, Parmar PN, Parikh YN, Kalathia IM. Study of umbilical cord blood culture in diagnosis of early-onset sepsis among newborns with high-risk factors. J Clin Neonatol. 2013;2(4):169-172. doi: 10.4103/2249-4847.123092

16. Kayange $N$, Kamugisha E, Mwizamholya DL, Jeremiah S, Mshana SE. Predictors of positive blood culture and deaths among neonates with suspected neonatal sepsis in a tertiary hospital, Mwanza-Tanzania. BMC Pediatr. 2010;10(1):39. doi: 10.1186/1471-2431-10-39
17. Chiabi A, Djoupomb M, Mah E, et al. The clinical and bacteriogical spectrum of neonatal sepsis in a tertiary hospital in Yaounde, Cameroon. Iran J Pediatr. 2011;21(4):441.

18. Chelliah A, Thyagarajan R, Katragadda R, Leela KV, Babu $\mathrm{RN}$. Isolation of MRSA, ESBL and AmpC- $\beta$-lactamases from neonatal sepsis at a tertiary care hospital. J Clin Diagn Res. 2014;8(6):DC24. doi: 10.7860/ JCDR/2014/8597.4512

19. Khanna A, Khanna M, Gill, M. ESBL Producing Gram negative bacteria-A cause of concern in neonatal septicemia in a tertiary care hospital. Int J Curr Microbiol App Sci. 2016;5(10):807-813. doi: 10.20546/ ijcmas.2016.510.087

20. Sivanandan S, Soraisham AS, Swarnam K. Choice and duration of antimicrobial therapy for neonatal sepsis and meningitis. Int J Pediatr. 2011;2011:712150. doi: 10.1155/2011/712150

21. Shrestha P, Das BK, Bhatta NK, et al. Clinical and bacteriological profiles of blood culture positive sepsis in newborns. J Nepal Paediatr. Soc 2007;27(2):64-67. doi: 10.3126/jnps.v27i2.1411

22. Femitha $\mathrm{P}$, Joy $\mathrm{R}$, Adhisivam B, et al. Candidemia in neonatal ICU-experience from a tertiary care hospital. Curr Pediatr Res. 2013;17(1).

23. Jin $Y$, Song $X$, Liu $Y$, et al. Characteristics of carbapenemase-producing Klebsiella pneumoniae as a cause of neonatal infection in Shandong, China. Exp Ther Med. 2017;13(3):1117-26. doi: 10.3892/ etm. 2017.4070

24. Falagas ME, Kasiakou SK, Saravolatz LD. Colistin: the revival of polymyxins for the management of multidrug-resistant gram-negative bacterial infections. Clin Infect Dis. 2005;40(9):1333-1341. doi: 10.1086/429323

25. Mehar V, Zade P, Joshi M, Rajput N, Bhatambar G. Neonatal ventriculitis with multi-drug resistant Acinetobactor baumanii: a case report and review of literature. Pediat Therapeut. 2012;2(5):131. doi: 10.4172/2161-0665.1000131

26. Hawley JS, Murray CK, Jorgensen JH. Development of colistin-dependent Acinetobacter baumanniiAcinetobacter calcoaceticus complex. Antimicrob Agents Chemother. 2007;51(12):4529-4530. doi: 10.1128/AAC.01115-07

27. Stoll BJ. Infections of the Neonatal Infant. In: Kliegman RM, Behrman RE, Jenson HB, Stanton BF (eds). Nelson Textbook of Pediatrics, 19th ed. Elsevier. 2011;103(2):629-648. doi: 10.1016/B978-1-43770755-7.00103-2

28. Abdel-Hady H, Hawas S, El-Daker M, El-Kady R. Extended-spectrum $\beta$-lactamase producing Klebsiella pneumoniae in neonatal intensive care unit. $J$ Perinatol. 2008:28(10):685-690. doi: 10.1038/jp.2008.73

29. Gray JW. Which factors predict hospital-acquired lateonset neonatal sepsis? Pediatr Health. 2008;2(4):477484. doi: 10.2217/17455111.2.4.477

30. Okada Y, Klein NJ, van Saene HK, Webb G, Holzel $\mathrm{H}$, Pierro A. Bactericidal activity against coagulasenegative staphylococci is impaired in infants receiving long-term parenteral nutrition. Ann Surg. 2000;231(2):276. doi: 10.1097/00000658-200002000- 


\section{8}

31. Mohammed D, El Seifi OS. Bacterial nosocomial infections in neo intensive care unit, Zagazig University Hospital, Egypt. Egypt Paediatr Assoc Gaz. 2014;62(34):72-79. doi: 10.1016/j.epag.2014.10.001

32. Kung $\mathrm{YH}, \mathrm{H}$ sieh $\mathrm{YF}$, Weng $\mathrm{YH}$, et al. Risk factors of late-onset neonatal sepsis in Taiwan: A matched casecontrol study. J Microbiol Immunol. 2016;49(3):430435. doi: 10.1016/j.jmii.2013.10.001

33. Babazono A, Kitajima H, Nishimaki S, et al. Risk factors for nosocomial infection in the neonatal intensive care unit by the Japanese Nosocomial Infection Surveillance (JANIS). Acta Med Okayama. 2008; 62(4):261-268.

34. Ruffell A, Adamcova L. Ventilator-associated pneumonia: prevention is better than cure. Nurs Crit Care. 2008;13(1):44-53. doi: 10.1111/j.14785153.2007.00248.x

35. Vijayakanthi N, Bahl D, Kaur N, Maria A, Dubey NK. Frequency and characteristics of infections caused by extended-spectrum beta-lactamase-producing organisms in neonates: a prospective cohort study. Biomed Res Int. 2013;2013:756209. pmid:24175299. doi: 10.1155/2013/756209
36. Jain A, Roy I, Gupta MK, Kumar M, Agarwal SK. Prevalence of ESBL producing gram negative bacteria in septicemic neonates in a tertiary care hospital. $J$ Med Microbiol. 2003;52(5):421-425. doi: 10.1099/ jmm.0.04966-0

37. Yusef D, Shalakhti T, Awad S, Khasawneh W. Clinical characteristics and epidemiology of sepsis in the neonatal intensive care unit in the era of multidrug resistant organisms: A retrospective review. Pediatr Neonatol. 2018;59(1):35-41. doi: 10.1016/j. pedneo.2017.06.001

38. Peirovifar A, Rezaee MA, Gharehbaghi MM. Prevalence of multidrug resistant extended-spectrum betalactamase producing Gram-negative bacteria in neonatal sepsis. Int J Women's Health Reprod Sci. 2014;2:138-45. doi: 10.15296/ijwhr.2014.21

39. Daef AE, Aly AS, El-Din ASS, El-Sherbiny NM, El-Gendy SM. Phenotypic and genotypic detection of extended spectrum beta-lactamase Klebsiella pneumoniae isolated from Intensive Care Units in Assuit University Hospital, Egypt. J Med Microbiol. 2009;18(2):29-40.

40. Shukla I, Tiwari R, Agrawal M. Prevalence of extended spectrum-lactamase producing Klebsiella pneumoniae in a tertiary care hospital. Indian J Med Microbiol. 2004;22(2):87. 